\title{
Cinéma et culte aux morts : fonction du rituel dans la construction du charisme politique (Espagne, 1936-1939)!
}

Vicente Sánchez-Biosca

\author{
FONDU AU NOIR
}

À l'aube du 20 novembre 1936 tombait sous les balles d'un peloton d'exécution, dans la prison d'Alicante, le fondateur de la Phalange espagnole, José Antonio Primo de Rivera. Ce même jour, dans la confusion d'une ville assiégée dont on craignait la chute imminente - Madrid - succombait, dans des circonstances encore obscures, Buenaventura Durruti, le libertaire mythique qui fit de Bujaraloz un royaume d'utopie. José Antonio et Durruti représentaient les extrémités d'un pays qui se déchirait alors dans une guerre d'extermination : l'un arborant l'esprit de la Révolution sociale, l'autre poussant l'accélérateur fasciste dans un contexte où la droite était par trop redevable à une tradition conservatrice. Deux projets en somme qui, dans la vie symbolique, pointaient bien au-delà du dénouement de la guerre et qui contribuèrent à faire rayonner ces deux figures dans l'horizon.

Certes, ils n'étaient pas les premiers tombés célèbres que le conflit générait ; ils l'étaient encore moins dans le vertige sacrificiel des mouvements politiques et sociaux qui cherchaient à se légitimer par le sang versé. Cependant, leur mort fut à l'origine d'un culte que la Première Guerre mondiale avait revêtu d'une dimension nationale et que les années 1920 orientèrent vers une martyrologie missionnaire dont le cas du SA Horst Wessel fut

1. Ce texte a ćté conçu dans le cadre du projet de recherche espagnol HAR 201232593. 


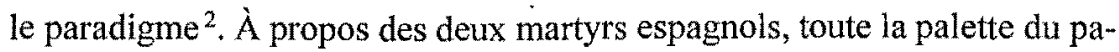
thos fut employée; la veine la plus exaltée du rapport à la mort s'activa et le culte aux disparus fut mis au compte d'une gestion du pouvoir entre les vivants avec qui ces héros entretenaient des échanges symboliques depuis leurs tombes. Incarnant deux projets politiques en temps de guerre, José Antonio et Durruti devinrent après leur disparition physique les emblèmes d'une éclatante puissance commémorative, puissance dont l'ancrage se place au cœur même de leur enterrement.

Les médias de l'époque furent conscients de ce qui se jouait dans l'opération, et la mise en scène des cortèges funèbres fut non seulement enregistrée par le cinéma, la photographie, la radio et la presse illustrée, mais conçue par l'entremise de ces instruments de propagande pour en agrandir le retentissement. Ainsi, le traitement du cinéma met à nu deux formes de liturgie et deux styles de charisme politique dont le motif premier est la cérémonie mortuaire et dont l'enjeu est la nécessité urgente de la transcender. Le héros tombé émerge alors dans une atmosphère de deuil qui forme avec la reprise de l'action un hiatus dont l'enjeu est le refus de se laisser inonder par l'océan de la mélancolie, la force de rédemption étant censée surgir du cérémonial lui-même. Un mot condense toute l'ambiguîté de ce leitmotiv qui est au cour des religions politiques : la ferveur. Car l'efficacité symbolique (pour emprunter l'expression à Lévi-Strauss) transforme les mots prononcés et les rôles joués en actes de manière à renforcer la cohésion du groupe. Plus importante se révèle la pénurie d'images cinématographiques disponibles de José Antonio et de Durruti, condition qui contraint les propagandistes à édifier le mythe avec du bricolage (photogrammes 1 et 2).

2. Horst Wessel était un martyr provenant du Kampzeit (période de combat). Voir CASQUETE, J., " "Sobre tumbas, pero avanzamos"; Horst Wessel y el troquel martirial en el nacionalsocialismo ", dans CASQUETE, J. et CRUZ, R. (dir.), Políticas de la muerte. Usos y abusos del ritual fúnebre en la Europa del siglo XX, Madrid, Catarata, 2009, p. 171-213. 

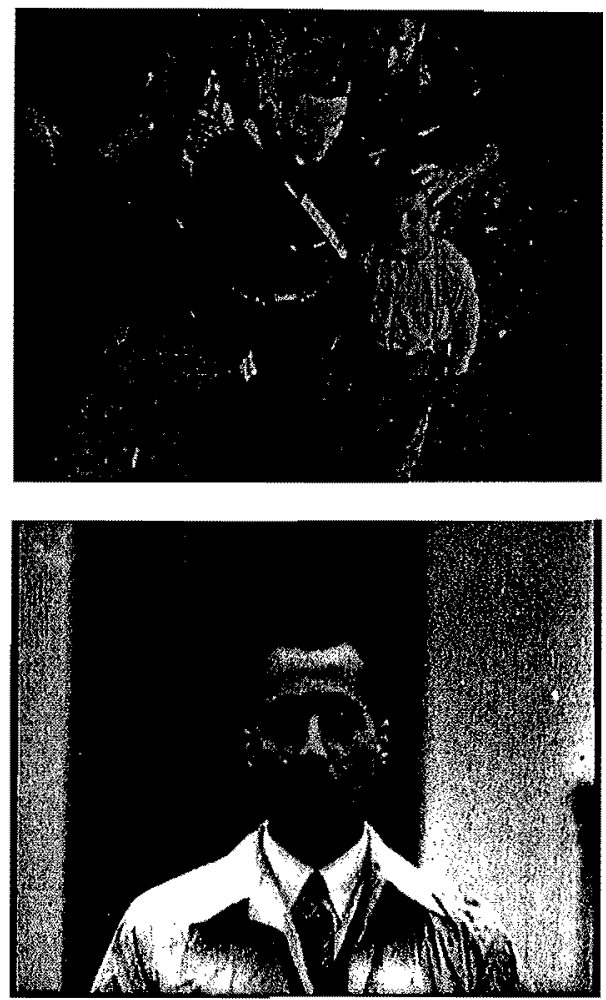

\section{DURRUTI EN DEUX VOLETS}

À quatre heures du matin le 20 novembre, dans la chambre numéro 15 de l'Hôtel Ritz de Madrid décédait Durruti. Cependant, son corps devait retourner à la scène privilégiée de ses exploits, Barcelone, où cette légende composite du bandit généreux et du terroriste imperturbable avait grandi. Le 23 novembre, après avoir traversé Alicante, Valence et Tarragone, le cortège quitta le siège de la CNT dans la capitale catalane s'acheminant, au milieu d'une foule immense, vers le cimetière de Montjuïc ${ }^{3}$.

Deux documents cinématographiques fixèrent l'événement, symbolisant les contradictions épineuses de la conjoncture de guerre à Barcelone.

3. PAZ, A., Durruti en la Revolución española, Madrid, Fundación Anselmo Lorenzo, 1996, chapitres 21 et 22 . Voir aussi la mosaïque de témoignages recueillis par ENZENSBERger, H. M., Der kurze Sommer der Anarchie. Buenaventura Dumut Leben und Tod, Francfort, Suhrkamp, 1972, chap. 7. 
L'enterrament de Durruti, film produit par Laia Films, entreprise rattachée au Commisșariat de Propagande de la Généralité de Catalogne, exprima le culte au héros avec le commentaire enlevé de son plus haut responsable, Jaume Miravitlles. The Mass Tribute to Buenaventura Durruti fut produit par la CNT et diffusé en deux versions, l'une espagnole et l'autre anglaise. Aucun de ces films ne transmet l'impression de chaos qu'évoquent les récits des témoins de l'époque, pour lesquels l'enterrement aurait été l'expression fascinante d'une ville bouleversée par la Révolution sociale ${ }^{4}$. Le récit de Kaminski le formulait avec une netteté non dépourvue d'exotisme : "Non, s'écrit-il, ce n'étaient pas des funérailles royales, c'étaient des funérailles populaires. Rien n'y était ordonné, tout se passait spontanément, à l'improviste. C'étaient des funérailles anarchistes, voilà leur majesté ${ }^{5} !$ \%. Il ajoutait :

«C'était une scène tragique et grotesque à la fois. Goya l'aurait dessinée, elle aurait trouvé place dans son cuvre. Je la décris telle que je l'ai vue, car je crois qu'elle laissait entrevoir les abîmes de l'âme espagnole. La mort en ce pays n'est ni un squelette horrible ni un bel adolescent qui porte une torche renversée. Elle est plutôt un bon ami, un camarade, un ouvrier... ${ }^{6}, \eta$

Pour étonnant que cela puisse paraître, les images de $L$ 'enterrament de Durruti ne confirment pas non plus de déchirure tragique. Bien au contraire, la foule nombreuse se mêle volontiers aux officiels, à la tête desquels le montage présente le président catalan, Lluís Companys, le consul soviétique Antonov-Ovshenko, l'ancien compagnon de Durruti et alors ministre de Justice Juan García Oliver. Plus encore, un climat solennel s'impose et la cadence grave du montage véhicule le rythme de marche funèbre. En revanche, c'est la rhétorique enflammée de Miravitlles qui dresse le panégyrique du héros en parcourant ses exploits, le deuil de sa disparition et l'exemple qu'il représente pour l'avenir. Dans le texte prononcé, et grâce à la maestria de la parole, un étrange syncrétisme est proposé entre le leader anarchiste et le simple combattant antifasciste, entre le révolutionnaire utopique des collectivisations et le défenseur de la République sur le front de Madrid, entre l'immigrant castillan et la Catalogne fière de sa singularité. Par le biais de Durruti, le culte aux morts se conclut par une promesse de fidélité aux tombés. Or, le style ampoulé du film réussit à dissimuler certains

4. Dans l'épisode funèbre résonnent les mêmes accords légendaires qui suintent les mémoires de George Orwell, Franz Borkenau, Simone Weil et d'autres voyageurs de Barcelone dans les six demiers mois de 1936.

5. KAMINSKI, H. E., "Funérailles d'un héros ", Ceux de Barcelone [1937], Paris Allia, 1986, p. 46-48.

6. Ibid., p. 45. 
détails qui n'avaient pas échappé à l'observation de Kaminski, à savoir : l'absence de protocole qui régnait dans les rangs du cortège, les vêtements informels de la foule, l'esprit de camaraderie à l'égard du défunt, l'héterogénéité des participants. Ceux-ci sont justement les traits que la production libertaire met de son côté en avant, l'élément marquant étant une sorte de proximité, voire d'intimité, entre le filmant et le filmé. À la vision de ces prises de vues, on éprouve le sentiment d'un appareil complice qui stimule les regards caméra comme des échanges entre égaux ; singularité des reportages anarchistes dès le pionnier Reportage du mouvement révolutionnaire à Barcelone (Mateo Santos, 1936), dont la vision inspira Jean-Louis Comolli pour son film Buenaventura Durruti anarchiste (2000). Une caméra, chose surprenante en 1936, faisait partie de l'événement (photogramme 3).

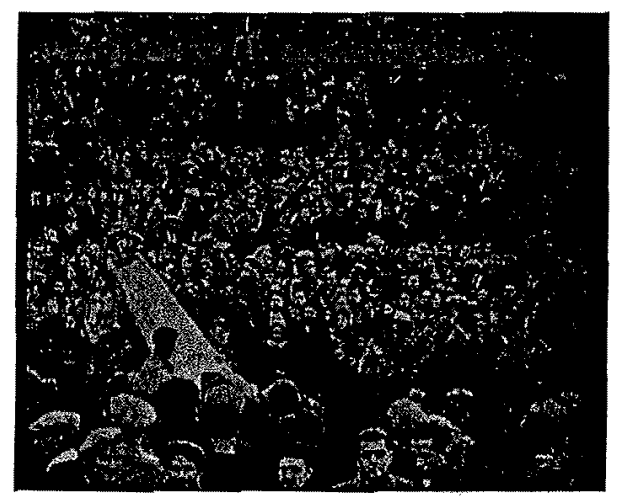

\section{SIX PIEDS SOUS TERRE, TU N'ES PAS MORT}

La coïncidence dans le temps des deux morts n'en contribue pas moins à saisir l'abîme qui les sépare, autant du point de vue de la liturgie que du charisme. La nouvelle de l'exécution de José Antonio fut diffusée aussitôt dans la presse républicaine et internationale, tandis qu'un mur de silence s'abattait sur l'Espagne nationaliste, prise dans un débat encore non résolu sur l'idéologie du soulèvement militaire de juillet 1936 - le commandement de l'armée n'avait pas encore réussi à faire table rase des conflits entre l'Église, les royalistes, les carlistes, les phalangistes et les autres obédiences. La question du charisme n'en taraudait pas moins les autorités militaires et politiques : Franco avait été investi Généralissime fin septembre 1936, mais son accession à la direction du parti unique ne se produirait qu'en avril 1937 lors du décret fusionnant les partis sous le nom de FET-JONS - le titre de chef de l'État et du gouvernement ne lui serait octroyé que le 30 janvier 
1938, suite à la constitution d'une structure d'État stricto sensu. Durant cette longue période, la mort du Fondateur de la Phalange resta secrète, placée sous un silence quasi sépulcral, José Antonio étant mentionné dans le discours du régime sous une formulation à la fois euphémique et paradoxale : le disparu d'un mouvement qui, à l'instar du fascisme italien, avait fait de l'appel aux tombés un cri de combat - Présent!-serait désormais évoqué comme l'Absent ${ }^{\text {? }}$. Au cours de deux ans de définition idéologique où Franco prit dans ses mains les rênes d'un pouvoir sans fissures, l'A bsent fut invoqué comme un espoir ou une sanction. En novembre 1938, la guerre touchant à sa fin, la mort de José Antonio fut enfin reconnue et les cérémonies in $a b$ sentia (le cadavre gisait encore en zone républicaine) furent les signes avantcoureurs du grand rattrapage sur le refoulement.

Une fois la victoire franquiste accomplie, et Franco transformé en leader incontournable des commémorations, défilés et bains de foules, le Fondateur fut associé au deuil en misant sur le côté morbide du culte aux morts, à deux différences près : d'une part, la religion politique dont il était le martyr s'imprégnait d'une forte auréole chrétienne et, d'autre part, le culte du héros mort ne se faisait pas au détriment de la gloire du vivant. Dans ce cadre et par l'initiative des intellectuels fascistes qui détenaient en 1939 les services de propagande, fut envisagé un des plus spectaculaires pèlerinages du XX $X^{\mathfrak{e}}$ siècle. À la fin du glacial mois de novembre 1939 , coïncidant avec la troisième commémoration de l'exécution de José Antonio, un itinéraire fut tracé pour transporter la dépouille du martyr de la prison d'Alicante jusqu'au panthéon de l'Escorial, lieu de mémoire bâti par Philippe II en mémoire de la victoire espagnole dans la bataille de Saint-Quentin (1557). Durant dix longues journées de marche, les membres de la vieille garde du parti marchèrent cinq cents kilomètres, bière à l'épaule, se relevant tous les cent mètres, parsemant le trajet d'innombrables célébrations. Le cortège atteignit sa destination le 30 novembre ${ }^{8}$.

Rien ne fut laissé au hasard : un minutieux scénario prit à son compte le délire liturgique, la structure du cortège, la surimposition de signes religieux et de symboles païens (les rites du feu, inspirés de la flamme sacrée

7. La Phalange s'inspirait de l'Appello fascista (rite d'appel), qui constituait le moment décisif où le nom du tombé était répondu par les chefs de squadra avec le mot iPresente! C'est ainsi que les rites mortuaires projetaient le sang des morts sur le sens mystique de la communion avee les vivants. Voir GENTLLE, E., Il culto del littorio : la sacralizzazione della politica nell' Italia fascista, Roma-Bari, Laterza, 1993 (Je cite l'édition espagnole, p. 54-56).

8. Gibson, I., En busca de José Antonio, Barcelone, Planeta, 1980, p. 248. L'auteur suggère que ce trajet aurait pu être inspiré par célui qu'en 1478 avait fait la dépouille de Philippe le Beau depuis les Flandres jusqu'à Grenade sous le regard attentif de Jeanne la Folle. 
des mythes aryens, éveilla des soupçons de la part des dignitaires ecclésiastiques) ${ }^{9}$. Poésie lyrique, architecture éphémère, chorégraphie des foules, sculpture, dessin, hymnes, banderoles, cris performatifs... tout fut mis en cuvre. La pièce décisive de cette campagne fut la production du film Présente ! En el enterramiento de José Antonio Primo de Rivera, seul document matriciel sur le culte aux morts jamais fabriqué par le fascisme espagnol $^{10}$.

Sur un ton de deuil, duquel participent les compositions plastiques et le montage de marche funèbre, s'étale la scène où l'on identifie les forces en présence : le clergé en tête exhibant la croix, la garde civile, les uniformes de la Phalange. Longeant le bord de la mer, le cortège fait s'immobiliser les marins qui, en contreplongée, comme des statues projetées sur le ciel, saluent bras levé. D'autres plans captent les reflets de la lumière matinale sur l'eau, qui semblent métaphoriser la beauté mélancolique qui s'empare des esprits. L'atmosphère, le port, la coloration émotionnelle, le héros mort : d'autres images cinématographiques se lisent ici, en creux, dont l'empreinte indélébile n'avait pas cessé de séduire les propagandistes espagnols : la fameuse séquence du Cuirassé Potemkine (S.-M. Eisenstein, 1925) où le corps de Vakoulintchouk exposé dans le port d'Odessa reçoit le dernier adieu de ses camarades et l'hommage du peuple. La « musique plastique " qu'Eisenstein évoqua à ce propos dans La non-indifférente nature ", la participation de la nature au drame (la séquence des brumes tournée par Édouard Tissé) et, surtout, l'esprit d'abattement qui règne autour du cadavre sont à l'origine de la citation par les cinéastes espagnols, qui avaient revendiqué l'efficacité du modèle eisensteinien ${ }^{12}$. Il n'en reste pas moins vrai que la dialectique entre intensité de la souffrance et déclenchement du processus révolutionnaire aura, chez Eisenstein, des conséquences bien différentes.

9. Pour le symbolisme du feu, Mosse, G. L., The Nationalization of the Masses. Political Symbolism and Mass Movements in Germany from the Napoleonic Wars through the Third Reich, Londres/Ithaca, Cornell University Press, 1975, Chap. 2. Quant aux plaintes de l'Église catholique contre l'aspect païen des célébrations, DI FEBO, G., «I riti nazionalcattolecesimo nella Spagna franchista. José Antonio Primo de Rivera e il culto dei caduti (1936-1939) », dans RidolfI, M. (dir.), Rituali civili. Storia nazionale e memorie pubbliche nell'Europa contemporanea, Roma, Gangemi, 2006, p. 189-202.

10. Pour l'analyse de ce film, SANCHEZ-BIOSCA, V., « The cinematic image of José Antonio Primo de Rivera: somewhere between a leader and a saint ", Screen, $n^{\circ} 50$, 2009, p. 318-333.

11. EISENSTEIN, S. M., La non-indifférente nature /2, CEuvres /4, Paris, U.G.E., 1978, p. 47.

12. MostaZA, B, «El cine como propaganda », Primer plano $\mathrm{n}^{\circ} 10,22-\mathrm{XII}-1940$, p. 3. Le film phalangiste Rojo y negro (C. Arévalo, 1942) irait plus loin encore en s'appropriant plusieurs plans de Potemkine pour en inverser la signification. 
Et voilà que le cortège poursuit son chemin en s'enfonçant dans une terre calcinée par la guerre. La composition photographique de certains plans montre un glissement de sources iconographiques, soulignant l'acharnement phalangiste à donner corps à un réalisme d'inspiration anthropologique : des figures typiques mais âpres de l'Espagne rurale, comme un paysan saluant accompagné de son âne sous un portail, ou un berger dans la plaine immense à côté de son troupeau, renvoient à une tradition que Terre sans pain ( $L$. Bunuel, 1933) avait mis en pièces sans complaisance peu de temps avant (photogramme 4). Au monastère de l'Escurial, l'inhumation fait office de mirage, José Antonio, prophète de l'Empire, fusionnant avec les gloires impériales du passé.

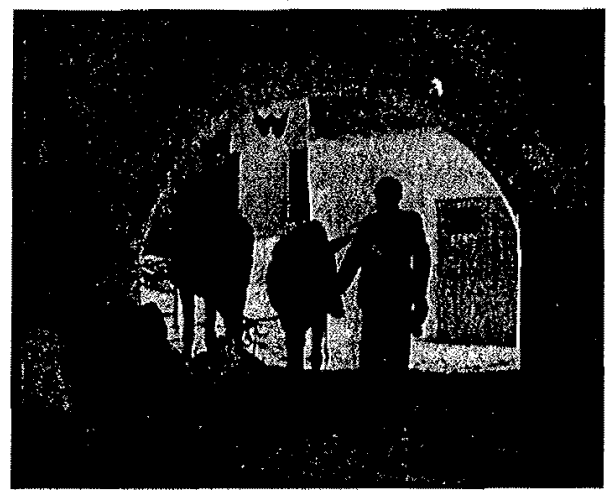

À ce point survient un détour inattendu : l'image nous transporte aux cieux où brillent les étoiles, métaphore phalangiste de la garde éternelle des tombés. De ce lieu céleste émerge en surimpression l'effigie de José Antonio qui descend un escalier, s'approche de la caméra et expose brièvement ses thèses politiques. Ces trois plans datent de 1935 et avaient été tournés pour les actualités Paramount. Or, placés à la fin du film, après la séquence de l'inhumation, le renversement chronologique suggère une véritable épiphanie du personnage, dont la parole n'est plus écoutée comme un programme, mais comme une prophétie faite par un revenant investi d'un pouvoir éternel. Sa résurrection nourrit l'aura de son image et de sa parole. À peine le discours fini, l'image de l'ange intouchable nous échappe, la caméra nous amenant à nouveau dans le firmament étoilé.

Une brève comparaison pourrait contribuer à évaluer la dimension surnaturelle dont il est question ici. À la différence de l'éclat magique de lumière qui baigne la paume de la main de Hitler lorsqu'il fait son passage triomphal au milieu de la foule de Nuremberg dans le Triomphe de la volonté (Leni Riefenstahl, 1935), face à la confrontation majestueuse des masses encadrées converties en constructions architecturales abstraites montées en 
champ-contrechamp avec leur leader, et à l'encontre de la démesure des symboles déployés par le paganisme nazi, phénomènes qui seraient à la base de la nouvelle volonté de puissance, Presente ! puise dans une symbolique religieuse d'inspiration baroque qui semble inséparable du fascisme espagnol et dont la portée se situe à une échelle plus humaine. En somme, ce documentaire de propagande joue sur un type de pathos trop mélancolique pour se transformer en action, mais ne reste pas moins étranger à l'apologie de la frénésie destructive prônée par Eisenstein, pour qui l'approfondissement d'une douleur sans fond est conditio sine qua non pour le déclenchement du vertige révolutionnaire. C'est ainsi qu'Eisenstein expliquait l'accomplissement dramatique du « saut qualitatif " d'Engels ${ }^{13}$.

\section{CULTE AUX MORTS ET RELIGIONS POLITIQUES}

Même si le culte aux morts remonte très loin dans la civilisation, sa mise au compte des concepts modernes de patrie et de nation est fortement soudée aux expériences de la Révolution française ${ }^{14}$. Au sein du complexe phénomène des religions politiques surgi de la désacralisation moderne, la Première Guerre mondiale joua un rôle d'honneur, réinvestissant ce que George Mosse nomma le «mythe de l'expérience de guerre ${ }^{15}$, dont l'un des plus significatifs exemples aurait été le long pèlerinage du soldat inconnu italien transporté en 1921 depuis Udine pour être inhumé dans l'autel de la patrie à Rome, le 4 novembre $1921^{16}$. Les mouvements fascistes, à forte composante nationaliste, prirent la relève en transformant les soldats tombés au front, après la prise du pouvoir, en martyrs du parti et de la nation. S'appuyant sur la puissance émotive de l'image, une telle mystique de la mort et un tel élan de rédemption étaient condamnés à trouver le cinéma sur leur chemin.

Revenons au cas espagnol. Soumise aux urgences de la guerre, l'expression prolongée du deuil risquait de sombrer dans un chagrin paralysant. Voilà pourquoi les enterrements de Durruti et de José Antonio se débattent dans

13. EisEnstein, S. M., « Le bond. Le passage de la quantité en qualité. Le passage daus son contraire ", La non-indifférente nature /l, Paris, U.G.E., 1976, p. 95.

14. MORO, R., "Rituales políticos / Religiones políticas ", dans CANAL, J. et MORENo LUzón, J. (dir.), Historia cultural de la política contemporánea, Madrid, CEPC, 2008, p. 97-147) articule, pour expliquer les religions politiques, les théries d'Albert Mathiez sur les cultures de la Révolution française, de Mare Bloch sur les rois thaumaturges et d'Enst Kantorowicz sur les deux corps du roi.

15. MossE, G. L., Fallen Soldiers. Reshaping the Memory of the World Wars, New York, Oxford, Oxford University Press, 1990.

16. GentiLe, E., Fascismo di pietra, Roma-Bari, Gius-Laterza, 2007, p. 5. 
une dialectique féroce qui oppose le deuil et la réanimation morale, dialectique qu'anarchistes et phalangistes gérèrent de manière bien distincte, autant dans la temporalité que dans la mise en scène, les mots d'ordre, ou même le rapport à la postérité ${ }^{17}$. Ces deux démarches en disent long sur les formes de liturgie politique agissantes à une conjoncture critique du $\mathrm{XX}^{\mathrm{e}}$ siècle.

Durruti et José Antonio n'ayant pu survivre à la guerre, il serait absurde de leur attribuer les conditions de vaincu et de vainqueur. Cependant, leur postérité n'en fut pas moins importante. Le rituel promu lors de l'enterrement de Durruti fait irruption dans le vif de la guerre, la suspend momentanément, mais l'adhésion anarchiste à la condition de camarade s'avère être un obstacle pour l'élévation du héros à un statut surhumain. Le vertige des combats finit par l'emporter. Par contre, José Antonio est enterré depuis trois ans lorsque sa commémoration est programmée avec toutes les ressources d'un État pris dans l'extase de la victoire et débarrassé des contraintes de la guerre. Le délire baroque, la présence morbide du cadavre et l'épiphanie du martyr montrent bel et bien une sanctification dont les phases ne comportent pas moins de fractures. Projets inachevés, les obsèques de Durruti et de José Antonio constituent à leur tour la première mise en scène d'une série de rituels où se tissent les imaginaires anarchistes et phalangistes.

\section{LE DERNIER VOL}

C'est ici que cette réverbération symbolique du 20 novembre devrait se terminer. Le franquisme ayant réussi à bâtir un immense mausolée conçu par Franco lui-même dès l'immédiat après-guerre, le corps de José Antonio fut à nouveau exhumé en 1959 pour y être conduit, cette fois-ci dans des circonstances plus discrètes, au Valle de los Caídos, nouveau lieu de mémoire qui était censé être sa tombe définitive. Or, deux décennies plus tard et presque quarante ans après la mort de José Antonio, alors que les cérémonies en son honneur avaient atteint une monotonie opiacée, ceux qui tiraient les faibles ficelles qui maintenaient Franco lié au monde décidèrent de relâcher la tension... et de le livrer à la mort. Ce fut le 20 novembre 1975. Le cadavre de Franco vint se placer face à la pierre tombale de José Antonio, dans le lieu si soigneusement choisi par le dictateur. Ce faisant, Franco aurait arraché à l'Absent son seul ancrage dans la vie symbolique : le lieu, la date, la commémoration. Et c'est peut-être ainsi que le deuxième corps de José Antonio se dissoudrait à jamais, comme Nosferatu, dans le soleil du matin.

17. Box, Z., España, año cero. La construcción simbólica del franquismo, Madrid, Alianza, 2010, Chap. 2. 


\section{Références bibliographiques}

Box, Z., España, año cero. La construcción simbólica del franquismo, Madrid, Alianza, 2010.

CASQUETE, J., "'Sobre tumbas, pero avanzamos' : Horst Wessel y el troquel martirial en el nacionalsocialismo $\%$, in CASQUETE, J. et CRUZ, R. (dir.), Politicas de la muerte. Usos y abusos del ritual fúnebre en la Europa del siglo XX, Madrid, Catarata, 2009, p. 171-213.

EISENSTEIN, S. M., La non-indifférente nature, CEuvres, Paris, U.G.E., 19761978.

ENZENSBERGER, H. M., Der kurze Sommer der Anarchie. Buenaventura Durruti Leben und Tod, Francfort, Suhrkamp, 1972.

DI FEBO, G., « I riti nazionalcattolecesimo nella Spagna franchista. José Antonio Primo de Rivera e il culto dei caduti (1936-1939) "), in RIDOLFI, M. (dir.), Rituali civili. Storia nazionale e memorie pubbliche nell'Europa contemporanea, Roma, Gangemi, 2006, p. 189-202.

GENTILE, E., Il culto del littorio. La sacralizzazione della politica nell 'Italia fascista, Roma-Bari, Gius-Laterza, 1993 [éd. espagnole, Buenos Aires, S. XXI, 2007].

GENTILE, E., Fascismo di pietra, Roma-Bari, Gius-Laterza, 2007.

GiBson, I., En busca de José Antonio, Barcelone, Planeta, 1980.

KAMINSKI, H. E., Ceux de Barcelone [1937], Paris, Allia, 1986.

MORO, R., «Rituales políticos / Religiones políticas », dans CANAL, J. et MORENO LuzÓN, J. (dir.), Historia cultural de la politica contemporánea, Madrid, CEPC, 2008, p. 97-147.

Mosse, G. L., The Nationalization of the Masses. Political Symbolism and Mass Movements in Germany from the Napoleonic Wars through the Third Reich, Londres, Ithaca, Cornell University Press, 1975.

Mosse, G. L., Fallen Soldiers. Reshaping the Memory of the World Wars, New York, Oxford, Oxford University Press, 1990.

MostazA, B., «El cine como propaganda », Primer plano, $\mathrm{n}^{\circ}$ 10, 22-12-1940. PAZ, A., Durruti en la Revolución española, Madrid, Fundación Anselmo Lorenzo, 1996.

SÁnCHEZ-BIosCA, V., «The Cinematic Image of José Antonio Primo de Rivera : Somewhere Between a Leader and a Saint $》$, Screen, $\mathrm{n}^{\circ} 50$, 2009 , p. 318-333. 


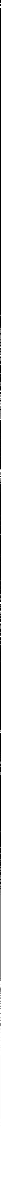

\section{Sous les images, la politique...}

Presse, cinéma, télévision, nouveaux médias ( $\mathrm{XX}^{\mathrm{e}}$-XXI $\mathrm{XIècle}^{\mathrm{e}}$

Sous la direction d'Isabelle Veyrat-Masson, Sébastien Denis et Claire Sécail 
Sous la direction de

Isabelle Veyrat-Masson, Sébastien Denis et Claire Sécail

\section{Sous les images, la politique...}

Presse, cinéma, télévision, nouveaux médias $\left(\mathrm{XX}^{\mathrm{e}}-\mathrm{XXI}^{\mathrm{e}}\right.$ siècle $)$ 
(C) CNRS Éditions, Paris, 2014

ISBN : 978-2-271-06988-7 


\section{Liste des contributeurs}

Nancy Berthier est professeur à l'Université Paris-Sorbonne.

Thierry Bonzon est maître de conférences à l'Université Paris-Est Marne-la-Vallée.

Alexandre Borrell est doctorant à l'Université d'Orléans.

Jérôme Bourdon est professeur à l'Université de Tel-Aviv (Israël).

Roland Cayrol est directeur de recherche associé au CEVIPOF (Sciences-Po), ancien directeur de l'institut CSA.

Jean-Paul Colleyn est directeur d'études à l'Ecole des Hautes Etudes en Sciences Sociales.

Marlène Coulomb-Gully est professeur à l'Université Toulouse 2 Le Mirail.

Jamil Dakhlia est professeur à l'Université Paris 3 - Sorbonne nouvelle.

Gaby David est doctorante à l'Ecole des Hautes Etudes en Sciences Sociales.

Sébastien Denis est maître de conférences HDR à l'Université d'AixMarseille.

Thierry Devars est doctorant et ATER à l'Université Paris-Sorbonne.

Jenifer Devresse est doctorante et assistante à l'Université de Liège (Belgique).

Jean-Pierre Esquénazi est professeur à l'Université Jean Moulin Lyon 3.

David Haigron est maître de conférences à l'Université Rennes 2.

Ramla Kronfol est docteur en études cinématographiques, spécialiste du cinéma du Moyen Orient.

Mireille Lalancette est professeure à l'Université du Québec à TroisRivières (Québec).

Frédéric Lambert est professeur à l'Institut Français de Presse de l'Université Panthéon-Assas.

Vincent Lemire est maître de conférences à l'Université Paris-Est Marne-la-Vallée.

Sarah Leperchey est maître de conférences à l'Université Paris 1 - Panthéon-Sorbonne. 
Joël Mak dit Mack est professeur de lettres et histoire au lycée de Thizy.

Marie Martin est maître de conférences à l'Université de Poitiers.

Martin O'Shaughnessy est professeur à Nottingham Trent University (Royaume-Uni).

Alain Peter est enseignant-chercheur au Centre universitaire d'enseignement du journalisme de l'Université de Strasbourg.

Cédrick Pinard est diplômé en communication sociale et agent d'information sportive à l'Université du Québec à Trois-Rivières (Québec).

Christian Poirier est professeur à l'Institut National de la Recherche Scientifique (Québec).

Olivier Pradervand est doctorant à l'Université de Lausanne (Suisse).

Vicente Sánchez-Biosca est professeur à l'Université de Valencia (Espagne).

Claire Sécail est chargée de recherche au Laboratoire Communication et Politique du CNRS.

Michael Temple est reader à Birkbeck College - University of London (Royaume-Uni).

Mélissa Thériault est professeure à l'Université du Québec à Trois-Rivières (Québec). Lille.

Erika Thomas est maître de conférences à l'Université catholique de

Héloïse Tillinac est docteur en science politique et consultante en communication.

Isabelle Veyrat Masson est directrice de recherche au CNRS, directrice du Laboratoire Communication et Politique.

Juliet Wagner est research assistant professor à Vanderbilt University (Etats-Unis). 